\title{
THE PREPARATION AND PROPERTIES OF THE o-AMINOPHENOL AZO-TUBERCULIN DERIVATIVE*
}

\author{
By HAJIME OKAMOTO \\ The Research Institute of Tuberculosis, Kanazawa University, Kanazawa \\ (Received for publication, November 16, 1949)
}

Since Koch's discovery of tuberculin, the active principle of tuberculin has long been the subject of many researchers, and a significant advance was made in the study of the nature of tuberculin when Seibert and Long and their associates $(1,2,3,4)$ succeeded in the isolation of the tuberculin active principle apparently in pure form, and as a result of which, as is well known, it has now become possible to compare accurately the tuberculin sensitiveness of human beings in the field testing.

Quite recently, working preliminarily with Maschmann's purified tuberculin $(5,6)$, and, following this, with Sauton's synthetic-medium Old Tuberculin, Ito and Koshimura of our laboratory have succeeded in establishing a simple procedure for preparing. $o$-aminophenol azo-tuberculin derivative quite equivalent in skin-reacting potency to the P. P. D. (purified tuberculin derivative) of Seibert. The new process is especially significant, in that it provides us with $o$-aminophenol azo-tuberculin preparations possessed of practically uniform skin-reacting potency in good quantities.

Although much work has been done in the research concerning the effect of chemical modification on the immunological reactivity of protein antigens $(7,8)$, there has, insofar as the writer is aware, been practically no work done towards the isolation of the active principle of tuberculin in the form of its azo-derivative.

In this paper I have tried to review the principal results of the research work which has been done in our Institute for these past two years.

\section{Basic Observations}

During the course of a systematic program devoted to the study of tuberculobacteriostatic power of $o$-aminophenol derivatives $(9,10,11,12)$, Ito and Koshimura (13), in 1947, discovered that $o$-aminophenol azo-tuberculin prepared by coupling the Maschmann's purified tuberculin with $o$-aminophenol showed no general-toxic effect upon the guinea-pigs infected with the human-type tubercle bacilli, while having powerful tuberculin skin-reacting potency comparable to the original purified tuberculin used.

On the other hand, they have noticed a curious phenomenon indicating that $\infty$-aminophenol azo-tuberculin having acquired the ability to dissolve the red blood cells (15).

The most theoretical importance to be noted here is the fact that the azotization

* The cost of this research has been defrayed from the Scientific Research Expenditure of the Department of Education. 
with $o$-aminophenol causes a marked dissociation between the general-toxic and skinreacting properties of tuberculin, the skin-reacting property being quite resistant to the introduction of $o$-aminophenol-azo radicals. Another important aspect of the diazotized tuberculin is that there is a good possibility that the skin testing can be made with the detoxified tuberculin.

On the basis of these fundamental observations extensive studies have been made with a view towards establishing procedures that could be applied for the preparation: of $o$-aminophenol azo-tuberculin derivative without using purified tuberculin as a starting: material.

\section{$o$-Aminophenol Azo-Tuberculin from Sauton's Synthetic-Medium $\mathrm{O}_{\text {Ld }}$ Tuberculin}

\section{Isolation and Purification Procedure}

Ito and Koshimura first found that for accomplishing the purpose, it is absolutely necessary to use a culture fluid of tubercle bacilli grown on non-protein synthetic media as the tuberculin sources, and that after azotization a precipitate occurs at $\mathrm{pH} 4.8-5.0$, which carries all of the skin-reacting activity. They then made a series of researches (14) to compare the relative quantity and potency of the products prepared by azotization with varying quantities of $o$-aminophenol per given amount of Sauton's syntheticmedium Old Tuberculin (or SOT in short), and found: (a) the yield of azo-products rised in proportion to the quantity of $o$-aminophenol used for the azotization of SOT, until, at the point of $1 \mathrm{~g} o$-aminophenol per $100 \mathrm{cc} \mathrm{SOT}$, practically all the active substance was transformed into its $o$-aminophenol-azo derivative; (b) all products obtained were found to be approximately equal potency in eliciting the specific skin reaction in tuberculous guinea-pigs, that is, the doses from $0.001 \mathrm{mg}$ to $0.000,1 \mathrm{mg}$ of O.A.-Azo-T.* preparations were equivalent to 0.1 " $\mathrm{mgm}$." of the original SOT ; (c) intraperitoneally in a salt solution of the products under consideration, the minimal lethal dose for tuberculous guinea-pigs laid between 3 and $5 \mathrm{mg}$, whereas the original SOT caused death of tuberculous animals when injected intraperitoneally in doses of 0.2 to $0.5 \mathrm{cc}$; (d) the: addition of too much quantity of diazotized $o$-aminophenol resulted in rather a reduction of the yield (22); (e) the hemolytic power of the resulting products rised with: increasing amount of diazotized $o$-aminophenol added to a given amount of SOT, having reached the maximum of hemolytic power at the ratio of $1 \mathrm{~g} o$-aminophenol to $100 \mathrm{cc}$. SOT, and the maximum of hemolytic titer generally falls between two separated dilutions of from $1: 20,000$ to $1: 50,000$ (22).

The possibility of purifying the active precipitate at its isoelectric point $(\mathrm{pH} \doteqdot 4.8)$; and method for eliminating adhering non-active extraneous materials were also carefully studied.

* The abbreviated nomenclature, O.A.-Azc-T., is occasionally employed to denote "o-Aminophenol Azo-Tuberculin derived from Sauton's synthetic-medium Old Tuberculin." 
On the other hand, the chemical phase of the phenomenon of the acquiring hemolytic property of proteins (including tuberculin) by coupling them with diazotized; $o$-aminophenol has been deeply studied by Koshimura (21), who has brought evidence that the hemolytic power possessed by O.A.-Azo-Protein derivatives is closely correlated with the content of $o$-aminophenol-azo radical.* And he added, "the finding of the new phenomenon seems to have important possibilities in the study for untangling fine structures of the protein molecules".

Thus, sufficient data, within a short time, have been accumulated to establish the essential steps leading to the preparation of highly purified $o$-aminophenol azo-tuberculin derivative from Sauton's synthetic-medium Old Tuberculin :

1. Growth of the human strain of tubercle bacillus on a Sauton's synthetic-medium.

2. Preparation from these cultures of a SOT (that is, Old Tuberculin from Sauton's synthetic-medium).

3. Azotization of tuberculin active principle present in SOT with $o$-aminophenoldiazonium chloride. (All manipulations in this process should be done at temperature lower than $5^{\circ} \mathrm{C}$.)

4. Precipitation of the active substance (that is, crude $o$-aminophenol azo-tuberculin: derivative) by acidification to $\mathrm{pH} 4.8$.

5. Removal of impurities from this crude O. A.-Azo-T. precipitate :

a) Washing with physiological salt solution. (Uncoupled protein substance, if any present, may be eliminated by this procedure.)

b) Repeating the purification procedure, in which the precipitate taken into solution with the aid of alkali and reprecipitated at the isoelectric point $(\mathrm{pH} \div 4.8)$ of the O. A.-Azo-T. derivative.

c) Treatment with alcohol, followed by dialysis. (Last traces of extraneous materials may be removed at these stages in the process.)

d) Washing with distilled water.

6. Complete drying of the O. A.-Azo-T. derivative in vacuo.

The method wich they recommended as most satisfactory and practical for the preparation of the O.A.-Azo-T. is presented in Table $1 .^{* *}$

* Modifying the Gibbs' test method for phenols, depending upon the quinone-chloroimide reaction producing indophenols (16), Koshimura and Koshiura have developed a colorimetrical method for the quantitative evaluation of 0 -aminophenol in body-fluids (17). This colorimetrical method was used for the estimation of $o$-aminophenol liberated from 0 -aminophenol azo-protein by boiling it with $\mathrm{SnCl}_{2}+\mathrm{HCl}$ solution.

* The formula of the Sauton's synthetic-medium :

\begin{tabular}{|c|c|}
\hline \\
\hline \multirow{2}{*}{ 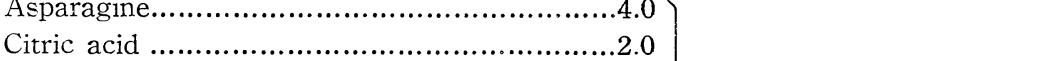 } & \\
\hline & \\
\hline Potassium monohydrogen phosphate..............05 & (Adjusted to $\mathrm{pH} 7.2$ with \\
\hline Magnesium sulfate ............................... 05 & \\
\hline Ferric ammonium citrate .......................... 0.05 & ammo \\
\hline Glycerol............................................ 60.0 & \\
\hline Distiiled water $\ldots . . . \ldots \ldots \ldots \ldots . . . .$. & \\
\hline
\end{tabular}


Table 1. Ito and Koshimura's procedure for preparing the 0 -aminophenol azo-tuberculin derivative from Sauton's synthetic medium Old Tuberculin.

Culture the human-type tubercule bacillus

$\left(\mathrm{H}_{2}\right.$-strain) on $800 \mathrm{cc}(200 \mathrm{cc}$ to a bottle) of

Sauton's synthetic medium for ten weeks at $37^{\circ} \mathrm{C}$.

$$
100^{\circ} \mathrm{C}, \stackrel{\downarrow}{60} \text { minutes }
$$

Filtrate through paper (weigh the bacterial bodies, after drying).

Concentrate the culture filtrate on a waterbath to a volume of $114 \mathrm{cc}$ (corresponds to $1 / 7$ of its original medium).

Filtrate through a Seitz E. K. Filter. (Preserve $10 \mathrm{cc}$ filtrate $\rightarrow$ for control purposes in SOT, $100 \mathrm{cc}$ biological experiments)

(Diazotize $1.0 \mathrm{~g}$ of $o$-aminophenol base, dis-) solved in $22 \mathrm{cc}$ of $\mathrm{N} / 1 \mathrm{HCl}$, with $0.63 \mathrm{~g}$ of sodium nitrite dissolved in $8 \mathrm{cc}$ distilled water at temperature of $0^{\circ}-5^{\circ} \mathrm{C}$, then add 50 cc chilled water, after testing excess of nitrite with starch iodide paper.

\section{Coupling}

Add the $o$-aminophenol-diazonium chloride solution just prepared to a cold mixture of $100 \mathrm{cc}$ SOT and $30 \mathrm{cc} \mathrm{N} / 1 \mathrm{NaOH}$, then keep weakly alkaline to litmus by adding $10 \mathrm{cc}$ $\mathrm{N} / 1 \mathrm{NaOH}$. Let stand overnight in ice-box.

Adjust to $\mathrm{pH} 4.8$, using $\mathrm{N} / 1 \mathrm{HCl}$, centrifuge (for 5 minutes at 3,000 R. P. M.) $\downarrow \rightarrow \begin{aligned} & \text { (Discard the clear, dark-brownish colored } \\ & \text { supernatant liquid) }\end{aligned}$

Wash the dark-brownish colored precipitate (crude O.A.-Azo-T.) by centrifugating three times, using $50 \mathrm{cc}$ of slightly acidified ( $\mathrm{pH} 5.0$ ) $0.85 \% \mathrm{NaCl}$ solution each time.

$$
\downarrow \text { (Discard the washings) }
$$

Dissolve the crude product in $20 \mathrm{cc}$ of salt solution by adding a minimal amount of N/10 $\mathrm{NaOH}$, filter off some insoluble material, and then precipitate the O.A.-Azo-T. by acidifying to $\mathrm{pH} 5.0$ with $\mathrm{N} / 10 \mathrm{HCl}$, centrifuge. Repeat resolution and precipitation more two times.

$\downarrow \rightarrow$ (Discard the supernatant liquid)

Dissolve the precipitate in $15 \mathrm{cc}$ of saline with the aid of a little $\mathrm{N} / 10 \mathrm{NaOH}$, then add $50 \mathrm{cc}$ of cold $60-70 \%$ alcohol and adjust to $\mathrm{pH} 4.8$, using $\mathrm{N} / 10 \mathrm{HCl}$. Let the suspension stand for about 10 minutes in the ice water, then thraw on the centrifuge.

$\downarrow \rightarrow$ (discard the deeply colored supernatant

Dissolve the precipitate in $20 \mathrm{cc}$ salt solution with a small amount of $\mathrm{N} / 10 \mathrm{NaOH}$.

$$
\downarrow
$$

Dialyse the O.A.-Azo-T. solution, in fish bladder membrane, against running water for 24 hours.

Filter through paper

$\downarrow$

Precipitate the O.A.-Azo-T. by $\mathrm{N} / 10 \mathrm{HCl}(\mathrm{pH}=4.8)$, centrifuge. $\downarrow \rightarrow$ supernatant liquid)

Wash the O.A.-Azo-T. two or three times, using $50 \mathrm{cc}$ of distilled water for each, centrifuge. $\downarrow \rightarrow$ (Discard the washings)

Place the Purified 0 -Aminophenol Azo-Tuberculin Derivative in vacuo.

(Yield : 150-200 mg)

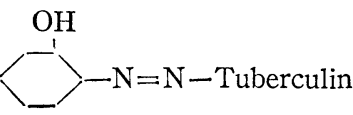


In Table II are summarized the results from experiments carried out upon seven different lots of " $\mathrm{H}_{3}$ " strain culture by carefully ensuring uniformity in all steps in" the process of preparation, except that age of culture varied from 63 to 82 days. The data in this table reveal two interesting points:

1) Variations in the luxuriance of growth on various lots do not make any appreciable difference in the potency of the final purified O. A.-Azo-T., but merely in the yield of that product; for example, the O. A.-Azo-T. prepared from the No. 1 lot, while present in only about one-forth the quantity, is nevertheless quite equal in skin-reacting potency per unit weight to the O. A.-Azo-T. derivatives prepared from other lots, Nos. $2-7$.

2) The yields varied from 185 to $286 \mathrm{mg}$ per liter of about ten-weeks original culture medium.

It may be therefore said that as long as the strain of tubercle bacillus, the growth of bacilli, the age of culture, the composition of the media, and the process of concentration, azotization, purification and finishing, remain the same, the O. A.-Azo-T. derivative, using $1 \mathrm{~g}$ of $o$-aminophenol for azotization of $100 \mathrm{cc}$ of SOT, can be produced with a high degree of uniformity in potency, in quantity and in nitrogen-content,* and that the length of the cultivation period must not be too short, if good yields are to be expected.

\section{Chemical and Physical Properties of the O. A.-Azo-T.}

The $o$-aminophenol azo-tuberculin derivative is a deeply brownish colored, amorphous powder. It is insoluble in water, but dissolves readily on the addition of a small amount of alkali. The total nitrogen content is about 10 per cent (see Table 2), and the content $\mathrm{OH}$

of $-\mathrm{N}=\mathrm{N}-$ radical appears to be from 4 to 6 per cent, calculated as $o$-aminophenol, which is liberated from O. A.-Azo-T. on $\mathrm{SnCl}_{2}+\mathrm{HCl}$ reduction (21). It is absolutely insoluble in iso-amyl alcohol, characterizing property which can be successfully used to distinguish the O.A.-Azo-T. from the brownish-colored substance which is formed in small amount as by-products from $o$-aminophenol-diazonium chloride itself.

O. A.-Azo-T. is precipitated by the usual protein-precipitating agents such as phosphotungstic acid, lead acetate, alcohol, hydrochloric, trichloroacetic, sulfosalicylic and tannic acids, and ammonium sulfate.

Owing to the deeply colored character of O. A.-Azo-T., its protein nature can not be successfully demonstrated by the color reaction method for the amino acids in proteins.

O. A.-Azo-T. does not diffuse through the fish bladder membrane.

* More recently, Ito and Koshimura found that $p$-aminophenol azo-tuberculin prepared under similar conditions from different SOT-lots of the same strain of tubercle bacillus is also possessed of a strong skin-reacting potency comparable to that of $\boldsymbol{o}$-aminophenol azo-tuberculin derivative.

At the same time, it was also pointed out that $p$-aminophenol azo-tuberculin is entirely different from O.A.-Azo-T. in having no hemolytic property. 


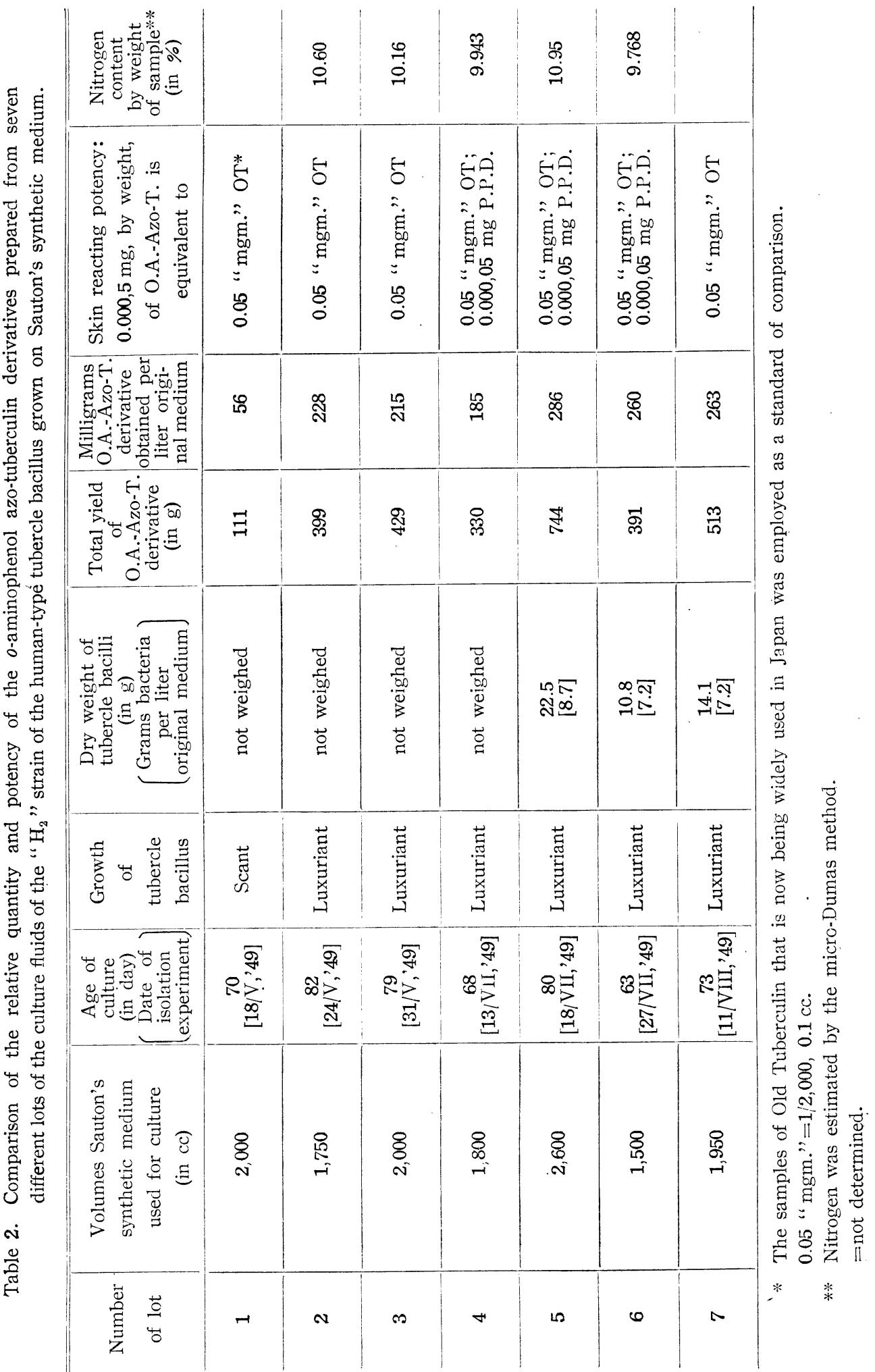


At $\mathrm{pH} 4.8$ the O. A.-Azo-T. is found to be completely out of solution.

Ito studied the effect of repeated purification manipulation upon the skin-tuberculin activity of O.A.-Azo-T., and noted neither increase nor decrease in its potency in the tuberculin reaction.

O. A.-Azo-T. in the dry state is stable indefinitely.

According to Sugibayashi and Takeuchi (18), boiling weak solutions of O. A.-Azo-T., such as solution of $1: 2$ millions, for 5 hours is without any effect upon its skin-reacting activity.

For practical use, O.A.-Azo-T. powder may be weighed and dissolved to any strength by suspending in saline and adding a small amount of $\mathrm{N} / 10 \mathrm{NaOH}$ necessary to give a clear solution. Dilution of $1: 2,000$ of $0.1 \%$ solution, containing $0.000,05 \mathrm{mg}$ of the dry powder in an injection dose of $0.1 \mathrm{cc}$, is entirely colorless, and if kept in a refrigerator at about $10^{\circ} \mathrm{C}$, the solution is serviceable for at least ten months.

\section{Tubercalin Skin-Reacting Potency of the O. A.-Azo-T.}

This phase of the work has been carried out under the direction of Prof. Dr. M. Kakishita, Director of the Bacteriological and Immunological Department of the Institute, by cooperation with Dr. K. Yuri and Dr. T. Shimao of the Kanazawa Municipal Hospital, who are all good experienced in this field (19). Preliminary trials of the potency of the $o$-aminophenol azo-tuberculin derived from Sauton's synthetic-medium Old Tuberculin were made on 11 apparently healthy adults on November 11, 1948, and evidence has been presented for the extremely high potency of O. A.-Azo-T. in eliciting tuberculin skin reaction without any unpleasant side effect.

Accordingly much large series of test was then carried out with $0.000,02-0.000,05$ mg O.A.-Azo-T. in comparison with 0.05 "mgm." $(1 / 2,000,0.1 \mathrm{cc})$ of OT (i. e. standard Old Tuberculin preparation in Japan. cf, Table 2).

1) Adults giving a strong skin reaction to OT likewise reacted strongly to O. A.Azo-T., and those with feable reaction to OT gave a weak reaction to O. A.-Azo-T.

2) Adults who reacted not to 0.05 "mgm." OT were also negative to $0.000,05 \mathrm{mg}$ O. A.-Azo-T.

Quite similar results were also obtained in skin-test experiments on school-children, who have no experience of the BCG. Needless to say, negative reaction to both these tuberculins indicates absence of natural infection with tubercle bacilli.

Thus, on a human-being skin-test-potency basis, they considered the dose of $0.000,05$ mg of O. A.-Azo-T. is equivalent to 0.5 " mgm." OT.

The results of comparative experiments performed with the P.P.D. and O.A.-Azo-T. 
were as follows: *

1) O. A.-Azo-T. was found to be, in fact, as potent as P. P. D.; for example, $0.000,05$ $\mathrm{mg}$, by weight, of O. A.-Azo-T. is equivalent in skin-reacting potency to $0.000,05 \mathrm{mg}$, by weight, of P.P.D.

2) In no case was there a reaction to P.P.D. and not to O. A.-Azo-T., and the reverse was also true.

Altogether 333 school-children, who had been inoculated with BCG, were tested with $0.000,05-0.000,1 \mathrm{mg}$ O. A.-Azo-T. in comparison with 0.05 "mgm." OT, and the investigators summarized the results in this kind of experiments as follows:

BCG-inoculated children who reacted not to OT were also negative to O. A.-Azo-T., whilst BCG-inoculated children reacting to OT were negative to O. A.-Azo-T., except that some cases (24 out of 333 probationers) were positive to both tuberculins. The groups who reacted positively to OT, but negatively to above-mentioned doses of $\mathrm{O}$. A.-Azo-T. were followed up by testing with more large strength of O. A.-Azo-T., until reaction was elicited, and it was found that as much as $0.005 \mathrm{mg}$ of O. A.-Azo-T. was. required to produce a positive tuberculin reaction. The fact that children who converted from negative to positive to 0.05 " mgm." OT after BCG-vaccination have a far lower degree of reactivity to O. A.-Azo-T. is especially noteworthy, for there is a possibility that BCG infection can be distinguished to some extent from the natural infection. On the other hand, it should also be noted that in 23 of 24 cases, who reacted not only to 0.5 “ mgm." OT but also to $0.000,05-0.000,1 \mathrm{mg}$ O.A.-Azo-T., signs of suspected tuberculous origin could be detected by X-ray examination of the chest. There is therefore: reason to suppose that these children have become infected with tuberculosis some: time after the $\mathrm{BCG}$ vaccination. If this interpretation in correct, the simultaneous; tests with OT and O. A.-Azo-T. may serve as a useful method for detecting primary infection occurring after the inoculation with BCG. Further work is needed.**

* P. P. D. :

One tablet of the First Test Strength (i. e. $0.000,1 \mathrm{mg}$ of P. P. D.) was dissolved in $0.5 \mathrm{cc}$ of the Buffered Diluent in the usual way. One tablet of the Second Test Strength (i. e. $0.025 \mathrm{mg}$ of P.P. D.) was dissolved in $0.5 \mathrm{cc}$ of the Buffered Diluent, and this "second test strength" solution was further diluted in sterile physiological salt solution in such dilution as to produce the required dose of $0.1 \mathrm{cc}$.

(The samples of P.P. D. were kindly furnished by American investigator for these tests.)

O.A.-Azo-T.:

$5.0 \mathrm{mg}$ of the O. A.-Azo-T. was dissolved in $5.0 \mathrm{cc}$ of sterile physiological salt solution with the aid of a minimal amount of $\mathrm{N} / 10 \mathrm{NaOH}$, and this original solution was then diluted in sterile physiological salt solution serially.

Skin Test:

The two tuberculins under comparison were simultaneously injected intradermally either on the fexor surface of the right forearm at an interval of $5 \mathrm{~cm}$ or into corresponding regions of the opposite forearms.

** It has recently been pointed out by Oyama that leucocytes obtained from guinea-pigs treated with O.A.-Azo-T. acquire an enhanced power to phagocytose killed tubercle bacilli.

In the prelimirary trials, Kura and Nakagawa have, however, failed to demonstrate that O. A.-Azo$\mathrm{T}$. has any action in preventing the experimental tuberculosis infection in guinea-pigs. 


\section{General Pharmacological Properties of the O. A.-Azo-T.}

The pharmacological properties of O. A.-Azo-T. have been studied by S. Fujita (20). His observations may be summarized as follows :

1) O.A.Azo-T. seems to be somewhat stronger in parameciumcidal action than does phenol.

2) It has a hemolytic property; the erythrocytes of rabbit, guinea-pig and human being are all equally susceptible to O. A.-Azo-T., having what appears from the in vitro experiments to be the minimum hemolytic concentrations of $1: 20,000$ to $1: 50,000$.

3) Intraperitoneally in an aqueous solution the M. L. D. for $16-19 \mathrm{~g}$ mice of O.A.Azo-T. is about $5 \mathrm{mg}$, and the toxicity of the agent is characterized by its delayed effect; however, while, the animal, received an intraperitoneal injection of the lethal dose of O.A.-Azo-T., remains seemingly normal for 3 to 5 days, a severe toxic signs leading to death appears thereafter. A single dose of $60 \mathrm{mg}$ O. A.-Azo-T. given intraperitoneally proved non-toxic to normal guinea-pigs.

4) Action on the isolated perfused blood vessels of frogs and guinea-pigs: The blood vessels of tuberculous guinea-pigs as well as those of normal animals were not significantly affected by O. A.-Azo-T.

5) Action on the isolated heart of frogs: O.A.-Azo-T., in dilutions of 1:5,000 to $1: 10,000$, produces a slight decrease in heart action.

6) Action upon the guinea-pig's intestine suspended in the Tyrode's solution: 0 . A.Azo-T., up to a dilution of $1: 100,000$, causes decrease of peristalsis and relaxation of the intestine of normal guinea-pigs.

Quite similarly, the movements of the intestine of tuberculous guinea-pigs were inhibited by the agent; no signs of Schutz-Dale's effect were ever observed with O. A.Azo-T.

From these experiments, it may, at least, be said that O. A.-Azo-T. preparation is quite free from histamine-like contaminants.

(It was further demonstrated that the action of SOT (OT and concentrate of glycerol-broth also), in contrast to O. A.-Azo-T., is generally followed by slight stimulation of the intestine.)

7) When O. A-Azo-T., was injected intravenously into rabbits (2-3 kg of bodyweight) in 10 to $20 \mathrm{mg}$ doses it caused neither appreciable despression in blood pressure nor change in respiratory movement.

Thus, the pharmacological and toxicological data just described also enable us to assess the absolute safety of O.A.-Azo-T. derivative for practical use in tuberculin skin testing in human being.

In passing, it should be here noted that O.A.-Azo-T. was found to be almost lacking in tuberculo-bacteriostatic activity in the Kirchner's medium containing serum. 
The writer is greatly indebted to Prof. Dr. N. Ishizaki, President of the College of Medicine, Kanazawa University, for earnest support in this work.

The writer wish to express his sincere appreciation to Dr. A. W. Bernheimer and Dr. W. L. Barksdale, New York University College of Medicine, for encouragement and advice.

\section{References}

1) Seibert, F. B., Aronson, J. D., Reichel, J., Clark, L. T., Long, E. R. and White, W. C.: Purified protein derivative, A standardized tuberculin for uniformity in diagnosis and epidemiology. Am. Rev. Tuberc., 30, 705, 1934.

2) Seibert, F. B.: The chemistry of tuberculin. Chem. Rev. 34, 107, 1944.

3) Seibert, F. B.: The isolation of three different proteins and two polysaccharides from tuberculin by alcohol fractionation. Their chemical and biological properties. Am. Rev. Tuberc., 59, 86, 1949.

4) Wells, H. G. and Long, E. R.: The Chemistry of Tuberculosis, second edition, 6, 93-113, 1932.

5) Maschmann, E. and Kuster, E.: Ueber die Reinigung des Tuberkulins. Z. physiol. Chem., 193, 215, 1930.

6) Maschmann, E. and Kuster, E. : Ueber die Gewinnung und Reinigung von Tuberkulin. Z.f. Tbk., 59, 225, 1931.

7) Landsteiner, K. and Lampl, H.: Ueber die Antigeneigenschaften von Azoprotein. Z. Immun.forsch., 26, 293, 1917.

8) Marrack, J. R.: The Chemistry of Antigens and Antibodies. 1938.

9) Okamoto, H.: Experimental studies in chemotherapy of tuberculosis. Annual Report of the Research Institute of Tuberculosis, Kanazawa Medical University. Vol. 4, 1946 and Vol. 6, 1948.

10) Okamoto, H. : Experiments on the chemotherapeutic effect of 0 -aminophenol upon tubercle bacilli.

- Jap. Med. J., 1, 422, 1948.

11) Sagara, S., Takamori, M. and Ito, R.: Some comparative experiments on the effect of streptomycin and $o$-aminophenol upon the tubercle bacillus. Jap. Med. J., 2, 119, 1949.

12). Sagara, S., Takamori, M., Ito, R., Koshimura, S. and Koshiura, R. : A comparative tuberculobacteriostatic study of $o$-aminophenol, $p$-aminosalicylic acid and 5 -aminosalicylic acid. Jap. Med. J., 2, 195, 1949.

13) Ito, R. and Koshimura, S.: Experimental study on the influence of azo-tuberculin upon guineapigs infected with tubercle bacilli. (Preliminary report) Jap. Med. J., 1, 427, 1948.

14) Ito, R. and Koshimura, S.: Experimental study on the influence of azo-tuberculin upon guineapigs infected with tubercle bacilli. Part II. Isolation of the 0 -aminophenol azo-tuberculin derivative from "Sautoṇ's synthetic-medium Old Tuberculin." Jap. Med. J., 2, 185, 1949.

15) Ito, R., Koshimura, S. and Fujita, S.: On the hemolytic action of 0 -aminophenol azo-protein delivatives. Jap. Med. J., 2, 130, 1949.

16) Gibbs, H. D.: Phenol tests. III. The indophenol test. J. Biol. Chem., 72, 649, 1927.

17) Koshimura, S. and Koshiura, R. : Eundamental studies in chemotherapy of tuberculosis. Part XXVI. Study on the method for the determination of 0 -aminophenol in blood and other body fluids. A colorimentrical method based upon indophenol reaction. Annual Report of the Reseach Institute of Tuberculosis, Kanazawa Medical University. VII, 1948.

18) Sugibayashi, A. and Takeuchi, H.: On the thermo-stability of 0 -aminophenol azo-tuberculin derivative in aqueous solution. Ibid., VIII, 1949 (in press).

19) Yuri, K.: Comparative skin test experiments with 0 -aminophenol azo-tuberculin derivative and Old Tuberculin in human being. Ibid., VIII, 1949. (in press)

20) Fujita, S.: Pharmacological studies of 0 -aminophenol azo-tuberculin derivative. Ibid., VIII, 1949. (in press)

'21) Koshimura, S.: Chemical studies on the 0 -aminophenol azo-protein derivatives. Reading at the meeting of the Japanese Pharmaceutical Association at Tokyo, July 7, 1649.

22) Ito, R. and Koshimura, S.: Unpublished observations. 